\title{
Uma metodologia para avaliar e ampliar o conhecimento de adolescentes do ensino fundamental sobre acidentes de trabalho
}

\author{
A methodology to evaluate and expand \\ knowledge on work-related accidents among \\ adolescents enrolled in elementary school
}

Maria Imaculada Medina Lima 1

Volney de Magalhães Câmara 1

\footnotetext{
1 Núcleo de Estudos de Saúde Coletiva, Hospital Universitário Clementino Fraga Filho, Universidade Federal do Rio de Janeiro. Av. Brigadeiro Trompowsky s/no, 5o andar, Ala Sul, Rio de Janeiro, $R J$ 21941-590, Brasil. medina@nesc.ufrj.br volney@nesc.ufrj.br
}

\begin{abstract}
Knowledge concerning work-related hazards and accidents among adolescents enrolled in an elementary public school was evaluated through an analysis of their occupational profile and discussions concerning concepts of risk situations at work and individual and collective measures for accident prevention and control. The results included a significant change in their understanding of work-related accidents as identified by statistical tests comparing the proportion of correct answers in a questionnaire applied prior to and at two moments after the intervention.

Key words Adolescence; Occupational Accidents; Education

Resumo O objetivo deste estudo foi analisar o entendimento dos adolescentes de uma rede pública de ensino fundamental sobre os riscos à saúde provocados pelo trabalho. Procurou-se traçar o perfil ocupacional dos adolescentes, discutir conceitos de acidentes de trabalho e situações de riscos, bem como, medidas individuais e coletivas de prevenção e controle de acidentes. $O$ resultado do estudo permitiu avaliar uma significativa mudança no entendimento dos conceitos de acidentes de trabalho através de testes estatísticos que compararam o índice de respostas corretas em três períodos cronológicos.

Palavras-chave Adolescência; Acidentes de Trabalho; Educação
\end{abstract}




\section{Introdução}

Ao se pensar na diversidade dos problemas de saúde gerados pelos processos produtivos, emerge a necessidade de experiências que busquem a compreensão dos agravos à saúde provocados pelo trabalho em grupos sociais específicos. A velocidade das mudanças desses processos produtivos implica o surgimento de situações de risco, em virtude da implantação de novas tecnologias e formas de organização do trabalho, e, como conseqüência, uma mudança no padrão de saúde da classe trabalhadora. Nesse contexto, os acidentes de trabalho são expressões da dinâmica dos processos produtivos, fazendo parte da vida cotidiana dos trabalhadores.

Entende-se que é possível criar mecanismos e alternativas que viabilizem uma discussão sobre os impactos do trabalho na saúde dos trabalhadores com grupos sociais específicos, aqui percebidos como sujeitos na dinâmica dos processos produtivos. Portanto, o ambiente de trabalho é objeto de conhecimento e intervenção numa perspectiva preventiva.

Os indicadores existentes, especialmente os de mortalidade e letalidade, evidenciam que as condições de trabalho no Brasil são preocupantes. Dados do Instituto Nacional de Seguridade Social demonstram que, entre 1985 e 1994, a diminuição da taxa de incidência acidentes de trabalho $(63,97 \%)$ não foi acompanhada por um decréscimo da proporção de mortes por esses acidentes. Ao contrário, a taxa de letalidade aumentou $98,28 \%$ no mesmo período (Tambellini \& Câmara, 1998), o que sugere a possibilidade de estar ocorrendo subnotificação dos acidentes de menor gravidade no Brasil.

Pode-se supor que os dados de acidentes com a população trabalhadora são maiores do que os registrados oficialmente, se levarmos em consideração a restrição de ordem quantitativa, pois essas informações são colhidas nas Comunicações de Acidentes de Trabalho (CATs), portanto, somente dos trabalhadores com vínculo empregatício formal. Outro viés de informação é observado na autorização dada às empresas para notificação e atendimento ao acidentado e a responsabilidade do pagamento dos primeiros 15 dias, o que sugere ocultação de acidentes mais leves. Fora desse quadro, há também um grande contingente da população que está no mercado informal, notadamente as crianças trabalhadoras. Gomez et al. (1992) comentam que os dados oficiais de acidentes de trabalho no Brasil são altamente questionáveis. Atribuem tal fato aos diagnósticos incorretos dos efeitos à saúde, bem como às deficiências do sistema de coleta de informações pelo setor público já apontadas.
No tocante aos registros das doenças causadas pelo trabalho, pode-se destacar a importância da implantação dos Programas de Saúde do Trabalhador nos últimos dez anos. Nesse período, ocorreu um crescimento do número das doenças ocupacionais notificadas no Brasil, indo de 5.217 casos no ano de 1990 para mais de 20.000 em 1995 (PAHO/WHO, 1998). Dias (1994) organizou um cadastro de 161 programas ou atividades indicadas como de saúde do trabalhador na rede pública, que possivelmente favoreceram o acesso dos trabalhadores a serviços que podem ter contribuído para a comprovação do nexo causal entre os processos de trabalho e a doença.

Quanto ao setor informal, a Organização Internacional do Trabalho (OIT) estima que o percentual de ocupados neste setor aumentou de 51,6\% em 1990 para 56,7\% em 1996 (OIT, 1997; PAHO/WHO, 1998). A OIT estima também, para a primeira metade dos anos 90 , um grande aumento do trabalho infantil em alguns países da América Latina em decorrência das condições sócio-econômicas, entre outros fatores condicionantes. Tomando-se o Brasil como exemplo, a evolução das taxas de participação laboral da população de 10 a 14 anos de idade, que em 1990 era de 17,5\%, passou para 20,4\% em 1995 (OIT, 1997).

Asmus et al. (1996) citam que as doses farmacológicas e terapêuticas das diferentes substâncias químicas administradas em crianças e adolescentes são ajustadas por quilo de peso corporal, a fim de evitar efeitos tóxicos e overdo$s e$. Portanto, crianças e adolescentes expostos no trabalho a agentes químicos podem ser mais afetados do que os adultos expostos às mesmas concentrações destas substâncias, uma vez que os primeiros têm menor peso e maiores níveis de absorção por peso corporal.

Lima (1999) demonstrou, mediante a análise de estatísticas de acidentes de trabalho, que, das 3.284 mortes por esses eventos no Brasil, 361 $(11,0 \%)$ ocorreram em Minas Gerais. Quanto à população adolescente, o Estado contribuiu com um percentual de $21,9 \%$ do total de 105 mortes de trabalhadores até 19 anos, representando um coeficiente de 14,95 por 100.000 trabalhadores, maior que o encontrado em outros estados, como São Paulo $(5,36)$ e Rio de Janeiro $(3,18)$.

É, portanto, nessas condições, que surge a necessidade de se criar alguma forma de enfrentamento teórico e metodológico capaz de captar novos enfoques da relação saúde e trabalho. Significa estudar parcialmente essa relação por meio de estratégias que possibilitem uma discussão sobre o tema acidente de trabalho em um determinado grupo social, para poder suscitar novas formas de análise e intervenção. Ele- 
geu-se a escola como local para desenvolver este estudo por ser o centro institucional do processo de educar.

\section{Material e métodos}

O estudo foi realizado em duas escolas municipais do ensino fundamental do Município de Leopoldina, Estado de Minas Gerais, e baseouse em experiências anteriores do Núcleo de Estudos de Saúde Coletiva em desenvolver programas educativos. Tais programas demonstraram, através da discussão de situações de risco e conceitos de acidentes de trabalho, a evolução da assimilação desse conhecimento por meio de um questionário aplicado antes e depois do desenvolvimento do programa (Câmara et al., 2000).

Uma questão inovadora no presente estudo foi modificar o aspecto temporal da aplicação do questionário utilizado para avaliar a assimilação dos conceitos de acidentes de trabalho. Enquanto nas experiências anteriores analisou-se a variação do conhecimento em uma semana, este estudo procurou avaliar as variações do conhecimento adquirido em três momentos, sendo o terceiro quatro meses depois.

A metodologia foi constituída de seis etapas, seguindo uma ordem cronológica, sendo as cinco primeiras durante uma semana e a sexta etapa quatro meses depois, a saber:

a) Apresentação dos pesquisadores aos escolares e explicação das atividades a serem realizadas. Em seguida, foi solicitado aos adolescentes que respondessem ao questionário, elaborado com perguntas simples e diretas, que caracterizam o perfil ocupacional, situação de moradia, sexo, idade, apresentando em seu conteúdo situações de vida e trabalho para serem relacionadas com acidentes e doenças do trabalho. Esta etapa incluiu não só a projeção de diapositivos retratando algumas ocupações, para que os adolescentes pudessem, através das imagens, interpretar as mesmas do ponto de vista de seu impacto na saúde, como também a discussão dos conceitos de acidente de trabalho adotados pela legislação brasileira.

b) Discussão sobre profissões escolhidas por eles para avaliar seus impactos na saúde. Para motivar a discussão, foi sugerida a escolha de suas próprias ocupações (se fosse o caso), assim como as de seus pais, parentes próximos, amigos etc.

c) Planejamento de atividades práticas a serem realizadas pelos adolescentes para abordagem do tema saúde e trabalho, sendo oferecido apoio logístico para desenvolvimento das mesmas. d) Desenvolvimento das atividades planejadas pelos adolescentes.

e) Avaliação final pelos adolescentes do trabalho desenvolvido. Também foi aplicado o mesmo questionário para verificar os índices de acertos e erros dos conceitos trabalhados, bem como para analisar os demais dados contidos no questionário. Nessa fase, os adolescentes assistiram à projeção do vídeo produzido durante o desenvolvimento de todas as etapas.

f) Aplicação do questionário quatro meses depois, sem alteração em seu conteúdo, mas com modificações nas situações de vida e trabalho, para verificação de possíveis mudanças no entendimento dos conceitos.

Os dados obtidos por meio dos questionários aplicados nos três momentos foram digitados para compor um banco de dados e armazenados em microcomputadores, tendo como suporte o programa D-Base (Borland International, 1986). Para análise dos dados, utilizou-se o teste estatístico McNemar, apropriado para avaliar a significância das diferenças observadas nas respostas dos alunos nos três momentos em que responderam ao questionário (Fleiss, 1979).

\section{Resultados}

Participaram da metodologia educativa 66 adolescentes das 7a e 8a séries, porém, para a análise pareada dos questionários, foram incluídos 61 adolescentes que compareceram em todos os três momentos.

Dos 66 alunos, $65 \%$ residiam em área urbana e $35 \%$, em área rural. Como a região tem na pecuária, em pequenas propriedades, sua base econômica, muitos moram em pequenos distritos que são considerados zonas urbanas pela Fundação Instituto Brasileiro de Geografia e Estatística, mas que, na realidade, são margeados por propriedades rurais, o que sugere o vínculo de seus moradores a atividades desta natureza.

Quando à distribuição por sexo, 55\% eram do sexo feminino e $45 \%$, do sexo masculino. No tocante à idade, houve maior proporção dos que tinham 14 e 15 anos, correspondendo a $33 \%$ e $27 \%$ respectivamente. Por fim, no que se refere à situação ocupacional, $67 \%$ relataram não trabalhar. Depoimentos dos professores mostraram que a maioria dos alunos tem atividades regulares na zona rural, tais como, tocar o gado, tirar leite da vaca, roçar pasto, ajudar a família na plantação da lavoura. Os professores também relataram que essas atividades somente são consideradas como trabalho pelos adolescentes quando existe um vínculo empregatício formal. 
Para o desenvolvimento de uma dinâmica que levasse em conta o entendimento dos adolescentes sobre riscos ocupacionais, foram projetados diapositivos que representavam ocupações selecionadas pela equipe. Além de discussões sobre medidas de prevenção e controle para cada situação, ao serem projetadas as ocupações, registraram-se as seguintes associações entre saúde e trabalho: professor com alergia ao giz, estresse e varizes pelo fato de ficar muito tempo em pé; trabalhador rural com risco de intoxicação por agrotóxico e acidentes com animais, enxada e foice; comerciante com varizes por permanência em pé por longos períodos; empregada doméstica e catador de lixo com lesões na coluna vertebral por peso excessivo e postura do corpo inadequada; lixeiro com acidentes por materiais perfuro-cortantes e lesões da coluna pelo peso dos sacos de lixo; carpinteiro com lesões traumáticas e presença de corpos estranhos nos olhos pela manipulação de madeira.

A análise seguinte (Tabela 1 ) refere-se aos questionários aplicados nos três momentos cronológicos em que se avaliou a variação das respostas corretas, antes e após o desenvolvimento da metodologia.

a) Quanto ao acidente típico, já desde o primeiro momento ocorreu um grande percentual de respostas corretas (83\%). Porém, ao contrário do esperado, não há progressão no número de acertos após uma semana (logo após a discussão dos conceitos, segundo a legislação brasileira); ao inverso, ocorreu uma redução nesse percentual. Novamente e paradoxalmente, essas taxas voltam a se elevar após quatro meses (sem fornecimento de qualquer conhecimento específico adicional), chegando a $85 \%$.
O resultado não correspondeu ao esperado possivelmente em razão da própria natureza da pergunta considerada, sugestionando para a resposta mais correta, já que foi observado um alto índice de acertos desde o primeiro momento. Outra explicação poderia ser creditada à possibilidade de ocorrerem respostas ao acaso, sem conhecimento específico, uma vez que, daqueles cinqüenta que acertaram no momento inicial, sete passaram a errar tanto do primeiro para o segundo, quanto do primeiro para o terceiro momento, não havendo a progressão esperada na quantidade de acertos.

b) No que se refere ao acidente de trajeto, o padrão é menos conflitante, pois uma baixa percentagem de alunos respondeu corretamente já em uma primeira ocasião $(43,9 \%)$, proporção que aumentou significativamente na segunda semana (logo após aula expositiva), atingindo 95\% e mantendo-se este percentual de acerto após quatro meses, quando até se esperava alguma redução.

$\mathrm{Na}$ análise pareada, observou-se que, mesmo considerando que o número de opções para resposta era menor (duas), podendo haver maior percentagem de acertos com respostas ao acaso, a expressiva variação do número de acertos entre os momentos demonstra o aproveitamento do programa. Dos 27 adolescentes que haviam inicialmente respondido corretamente, $100 \%$ mantiveram o acerto e nenhum adolescente que inicialmente acertou errou no segundo momento. Já dos 34 que inicialmente erraram, somente três continuaram errando, enquanto 31 passaram a acertar.

A análise do terceiro momento demonstra valores semelhantes, pois, dos 27 alunos que responderam corretamente no início, 26 conti-

Tabela 1

Distribuição temporal de proporções de acertos sobre conceitos de acidentes de trabalho.

Município de Leopoldina, Minas Gerais, 1998.

\begin{tabular}{|c|c|c|c|c|c|c|}
\hline Momentos/Conceitos & 10 Momento & $\% 1$ & 2o Momento & $\% 2$ & 3o Momento & $\% 3$ \\
\hline Acidente típico & 55 & 83,3 & 50 & 82,0 & 52 & 85,2 \\
\hline Acidente de trajeto & 29 & 43,9 & 58 & 95,1 & 58 & 95,1 \\
\hline Doença do trabalho & 54 & 81,8 & 55 & 90,2 & 60 & 98,3 \\
\hline Agressão no trabalho & 4 & 6,0 & 59 & 96,7 & 52 & 85,2 \\
\hline
\end{tabular}

Acidente típico: p valor de 0,001 e $\chi^{2}$ McNemar de 29,03 do primeiro para o segundo momento e p valor de 0,001 e $\chi^{2}$ McNemar de 27,27 do primeiro para o terceiro momento. Acidente de trajeto: $\mathrm{p}$ valor de 0,001 e $\chi^{2} \mathrm{McNemar}$ de 29,03 do primeiro para o segundo momento e $p$ valor de 0,001 e $\chi^{2}$ McNemar de 27,27 do primeiro para o terceiro momento. Doença do trabalho: significância estatística quando se comparou o primeiro com o terceiro momento ( $p$ valor de 0,01 e $\chi^{2}$ McNemar de 7,11).

Agressão no trabalho: $p$ valor de 0,001 e $\chi^{2}$ McNemar de 54,02 do primeiro para o segundo momento e $p$ valor de 0,001 e $\chi^{2}$ McNemar de 47,02 do primeiro para o terceiro momento. 
nuaram acertando. A análise estatística mostrou diferença com alta significância ( $p$ valor de 0,001 e $\chi^{2}$ McNemar de 29,03 do primeiro para o segundo momento e p valor de 0,001 e $\chi^{2}$ McNemar de 27,27 do primeiro para o terceiro momento).

c) No tocante à caracterização de doença de trabalho aconteceu outro fato singular. Novamente voltou a ocorrer um alto percentual de acertos já desde a primeira ocasião (82\%), havendo progressiva elevação tanto do primeiro para o segundo momento, quanto do segundo para o terceiro momento, em que $98 \%$ dos alunos responderam de forma correta. Talvez o esperado poderia até ser uma pequena redução dos acertos no terceiro momento.

Pela análise pareada, o fato interessante é que, dos $83 \%$ que acertaram inicialmente, cerca de $10 \%$ passaram a errar no segundo momento, logo após a intervenção, voltando a acertar quatro meses após esta. No terceiro momento, dos que acertaram inicialmente, $100 \%$ voltaram a acertar. O teste empregado somente evidenciou significância estatística quando se comparou o primeiro com o terceiro momento ( $\mathrm{p}$ valor de 0,01 e $\chi^{2}$ McNemar de 7,11).

d) Com relação à agressão no trabalho, um número muito baixo de alunos respondeu corretamente no primeiro momento (6\%), ocorrendo significativa elevação no segundo momento (96\%) e havendo a esperada redução no percentual de acertos após um intervalo de quatro meses (85\%). A avaliação na fase inicial pode ser explicada pela própria natureza da pergunta, com difícil aceitação da resposta considerada mais correta. Isso porque, ao se tratar de agressão física no local de trabalho, o adolescente poderia não ter a percepção de sua relação com os acidentes de trabalho.

Por meio da análise pareada, pode-se observar o impacto positivo do programa, visto que no segundo momento, dos 58 alunos que inicialmente tinham respondido de forma incorreta, 56 passam a responder de forma adequada, ainda que contrariando uma idéia pré-concebida, enquanto dois resistem a essa mudança. Ocorreu fato quase idêntico no terceiro momento, apenas com valores menos expressivos, com manutenção da resposta incorreta de nove dos 58 que tinham inicialmente errado, na aceitação e fixação do conceito. As diferenças voltaram a ser altamente significantes entre o primeiro e o segundo e entre o primeiro e o terceiro momentos (p valor de 0,001 e $\chi^{2}$ McNemar de 54,02 do primeiro para o segundo momento e $\mathrm{p}$ valor de 0,001 e $\chi^{2}$ McNemar de 47,02 do primeiro para o terceiro momento).

\section{Conclusões}

$\mathrm{Na}$ analise do desenvolvimento do conjunto da metodologia, pode-se admitir que, mesmo considerando a complexidade dos elementos que atuam na conformação do entendimento da relação entre saúde e trabalho, essa questão, quando é discutida em qualquer espaço social - neste caso a escola, traduz sentido e significado.

Através do diálogo estabelecido junto aos adolescentes, categorias de análise, como o processo de trabalho e os acidentes de trabalho, expressaram significado ao ganhar concretude. Questões sobre os conceitos e métodos de prevenção e controle dos acidentes de trabalho ganharam visibilidade através das discussões originadas de experiências individuais. Um entendimento de que a realidade possui um valor considerando-se a percepção e a experiência que se tem dela. Assim, as situações de vida e trabalho ganham conteúdo, na medida em que se faz uma releitura do vivido, do experimentado, traduzindo em novos entendimentos da questão.

Ressalta-se, aqui, a análise das variações das respostas nos momentos temporais que revelaram a produção de conhecimento pelos adolescentes no que se refere aos conceitos trabalhados. Destaca-se também a aplicação do questionário no terceiro momento, quando foi observado que o fator tempo não demonstrou uma perda do conhecimento adquirido.

Pode-se concluir que o procedimento metodológico desenvolvido permitiu a socialização de um determinado conhecimento técnico, assim como valorizou o conhecimento e experiências dos adolescentes no mundo do trabalho. Entende-se que, por meio do diálogo, criaramse novas formas de entendimento da questão saúde e trabalho e, mais especificamente, dos acidentes de trabalho.

\section{Agradecimentos}

Projeto financiado pelo Conselho Nacional de Desenvolvimento Científico e Tecnológico (CNPq). 


\section{Referências}

ASMUS, C. F.; RUZANNY, M. H.; BARKER, R. S. L. \& MEIRELLES, Z. V., 1996. Riscos ocupacionais na infância e adolescência - Uma revisão. Jornal de Pediatria, 72:203-208.

BORLAND INTERNATIONAL, 1986. D-Base III. Scotts Valey: Borland International.

CÂMARA, V. M.; TAVARES, L. M. B.; FILHOTE, M. I. F.; MALM, O. \& PEREZ, M. A., 2000. A program for the control of indoor pollution by metallic mercury. Environmental Research, 83:110-116.

DIAS, E. C., 1994. A Atenção à Saúde dos Trabalhadores no Setor Saúde (SUS) no Brasil: Realidade, Fantasia ou Utopia? Tese de Doutorado, Campinas: Departamento de Medicina Preventiva, Universidade Estadual de Campinas.

FLEISS, S., 1979. Statistical Methods for Trates and Proportions. New York: John Wiley and Sons.

GOMEZ, C. M.; CARVALHO, S. M. T. \& PORTO, M. F. S., 1992. Por uma ecologia do trabalho. In: Saúde, Ambiente e Desenvolvimento (M. C. Leal, P. C. Sabroza, R. H. Rodriguez \& P. M. Buss, org.), pp. 79-97, São Paulo: Editora Hucitec/Rio de Janeiro: ABRASCO.
LIMA, M. I. M., 1999. Saúde e Trabalho no Ensino Fundamental: Construção de um Programa pelos Professores. Dissertação de Mestrado, Rio de Janeiro: Núcleo de Estudos de Saúde Coletiva, Universidade Federal do Rio de Janeiro.

OIT (Organización Internacional del Trabajo), 1997. International Programme of the Elimination of Child Labour. Las Formas más Intorelables de Trabajo Infantil. Geneva: OIT.

PAHO (Panamerican Health Organization)/WHO (World Health Organization), 1998. Health Conditions in the Americas. Washington, DC: $\mathrm{PAHO} / \mathrm{WHO}$.

TAMBELLINI, A. T. \& CÂMARA, V. M., 1998. A temática saúde e ambiente no processo de desenvolvimento do campo da saúde coletiva: Aspectos históricos, conceituais e metodológicos. Ciência e Saúde Coletiva, 3:47-59.

Recebido em 20 de setembro de 2000

Versão final reapresentada em 4 de abril de 2001 Aprovado em 27 de junho de 2001 